\title{
Calidad educativa con enfoque transversal, contribución a la educación integral del ingeniero
}

\section{Educational quality with a transversal approach, contribution to the integral education of the engineer}

\author{
MORENO-AGUILAR, Ma. Antonia†** \& SÁNCHEZ-LÓPEZ, Guillermina \\ Instituto Tecnológico de Puebla. Avenida Tecnológico 420, Maravillas, 72220 Puebla, Pue. \\ ID $1^{\mathrm{er}}$ Autor: Ma. Antonia, Moreno-Aguilar / ORC ID: 0000-0003-3587-9300, CVU CONACYT ID: 467317 \\ ID $1^{\text {er }}$ Coautor: Guillermina, Sánchez-López / ORC ID: 0000-0001-5866-9362, CVU CONACYT ID: 626579
}

DOI: $10.35429 /$ JOTE.2019.10.3.1.8

Recibido 10 de Octubre, 2019; Aceptado 28 de Diciembre, 2019

\begin{abstract}
Resumen
México enfrenta grandes problemas sociales como pobreza, corrupción, desigualdad de acceso a la salud y la educación, contaminación, inclusión de minorías, desempleo entre otros; esto ha generado un gran deterioro en los comportamientos de las personas en el mundo globalizado actual, caracterizado también por los cambios sociales, políticos, culturales, científicos, tecnológicos. En este contexto, la Educación Superior es determinante para el desarrollo económico de un país y factor indispensable en el crecimiento del ser humano y la sociedad; tiene una gran responsabilidad de contribuir a desarrollar en el alumno las capacidades necesarias para desarrollar aprendizaje continuo; no únicamente en su formación técnica intelectual, sino en el desarrollo de actitudes que le motiven a aprender; que favorezca su educación integral mediante la aplicación de competencias en las que se vean inmersos sus conocimientos, habilidades, actitudes y valores; atendiendo sus capacidades afectivas, motrices, de relaciones interpersonales y de inserción y actuación social. Este trabajo tiene como objetivo, contribuir en la mejorara de la calidad educativa, asumiendo la formación del Ingeniero a través de su desarrollo integral con un enfoque transversal; que prepare al estudiante para enfrentar los retos de esta sociedad tan cambiante. El método utilizado para este trabajo es el enfoque de sistemas y da continuidad al documento "La vinculación como medio para mejorar el desempeño profesional y lograr el desarrollo humano integral del ingeniero".
\end{abstract}

Calidad, Transversalidad, Formación integral

\begin{abstract}
Mexico faces major social problems such as poverty, corruption, inequality of access to health and education, pollution, inclusion of minorities, unemployment among others; this has generated a great deterioration in the behavior of people in the current globalized world, also characterized by social, political, cultural, scientific, and technological changes. In this context, Higher Education is decisive for the economic development of a country and an indispensable factor in the growth of the human being and society; has a great responsibility to contribute to develop in the student the necessary skills to develop continuous learning; not only in his intellectual technical training, but in the development of attitudes that motivate him to learn; that favors his integral education by means of the application of competitions in which his knowledge, abilities, attitudes and values are immersed; attending to their affective, motor, interpersonal relations and social insertion and acting capacities. The objective of this work is to contribute to the improvement of educational quality, assuming the Engineer's training through its integral development with a transversal focus; that prepares the student to face the challenges of this changing society. The method used for this work is the systems approach and gives continuity to the document "Linking as a means to improve professional performance and achieve the integral human development of the engineer".
\end{abstract}

Quality, Transversality, Integral training

Citación: MORENO-AGUILAR, Ma. Antonia \& SÁNCHEZ-LÓPEZ, Guillermina. Calidad educativa con enfoque transversal, contribución a la educación integral del ingeniero. Revista de Educación Técnica. 2019. 3-10: 1-8.

\footnotetext{
*Correspondencia al Autor (Correo Electrónico: Antonia.moreno@itpuebla.edu.mx)

$\dagger$ Investigador contribuyendo como primer autor.
} 


\section{Introducción}

Mejorar la calidad de la educación superior ha sido una prioridad de las políticas públicas desde hace mucho tiempo, pero los resultados son desiguales. El sistema de aseguramiento de la calidad es voluntario, complejo, fragmentado y costoso, además de carecer de transparencia y coherencia. Por otra parte, un número desconocido de programas de instituciones privadas operan fuera de dicho sistema. Desde el punto de vista económico, la finalidad de la educación no se limita a ser una inversión individual o rentable, el Estado requiere diferentes disciplinas para alcanzar un equilibrado en el desarrollo social del país.

Los organismos internacionales establecen un enlace con las políticas de Educación Superior en México; la Organización para la cooperación y el desarrollo económico (OECD) plasma en su Resumen Ejecutivo (2019) algunas recomendaciones que son clave para mejorar la calidad de la educación superior, para el desarrollo de las competencias y los conocimientos avanzados, que son fundamentales para las economías modernas.

Gracias a la educación superior, los estudiantes desarrollan competencias y conocimientos técnicos, profesionales y disciplinares específicos avanzados, así como competencias transversales que les cualifican para una variedad de ocupaciones laborales. Este mismo organismo, en su publicación "Hacia una reforma educativa en México", establece que, la mejora en la calidad educativa es una prioridad política y social en México, especialmente en años recientes, debido a las altas tasas de pobreza, la fuerte desigualdad y el aumento de la criminalidad. Por tal razón, el sistema educativo debe adaptarse para cumplir su misión esencial de preparar al alumno para el trabajo y para la vida, la formación integral de profesionales competentes, que no solo depende de los conocimientos y habilidades que se adquieren en los salones de clase sino de las convicciones, sentimientos y valores éticos que regulan la actuación profesional del egresado.
Así mismo; el documento $37 \quad \mathrm{C} / 4$ aprobado por la Conferencia General de la Organización de las Naciones Unidas para la Educación, la Ciencia y la Cultura (UNESCO) brinda a los Estados Miembros, la oportunidad de definir una visión estratégica común de la Organización para los próximos ocho años y el nuevo siglo. La Estrategia a Plazo Medio de la UNESCO para 2014-2021 establece que, la mitad de la población mundial tiene menos de 25 años. El porcentaje de jóvenes en los países en desarrollo aumentará hasta el $89,5 \%$ en 2025. Estos hombres y mujeres jóvenes tendrán que hacer frente a las consecuencias de una explotación no sostenible de la Tierra y de sus recursos. Los jóvenes representan un inmenso potencial de cambio: sin embargo, aspiran a contar con las oportunidades y los recursos educativos, científicos, culturales y de comunicación necesarios para su desarrollo personal, el acceso a empleos decentes, la participación cívica y el entendimiento mutuo.

Esta juventud representa un potencial para el cambio si conseguimos garantizarle el acceso a la educación y reforzar su participación en los procesos de decisión. Los jóvenes no son solo agentes del crecimiento económico, sino también una fuerza de transformación y progreso en la sociedad, donde la paz y el desarrollo equitativo y sostenible son los objetivos fundamentales.

Una encuesta realizada por el Centro de Investigación para el Desarrollo (CIDAC) descubrió carencias en las competencias relacionadas con la comunicación escrita en español y la comunicación oral en español e inglés entre los egresados de educación superior. A lo anterior cabe añadir que los empleadores manifestaron que los egresados tenían escasa capacidad de síntesis de la información y de razonamiento lógico, y no mostraban sentido de la responsabilidad ni proactividad (CIDAC, 2014).

La transversalidad se ha convertido en un instrumento articulador que permite interrelacionar, el sector educativo con la familia y la sociedad. 
En el mundo contemporáneo muchas instituciones han diseñado estrategias para la formación de valores en el estudiante, utilizando el instrumento de ejes transversales con el fin de dar un enfoque integrador a su currículo, brindar una formación integral y formular un fundamento ético al funcionamiento de la propia institución. Los ejes transversales tienen un carácter globalizante, porque atraviesan, vinculan $\mathrm{y}$ conectan muchas disciplinas del currículo, lo que significa que se convierten en instrumentos que recorren asignaturas y temas que cumplen el objetivo de tener visión de conjunto; esto no quiere decir que reste importancia a las disciplinas, sino que obliga a una revisión de las estrategias aplicadas tradicionalmente en el aula al incorporar al currículo en todos sus niveles, una educación significativa para el estudiante a partir de la conexión de dichas disciplinas con los problemas sociales, éticos y morales presentes en su entorno.

Los análisis más destacados de los Temas Transversales han sido propuestos por los autores González, y Moreno y Muñoz. (1997), retoma los planteamientos de estos autores y agrupa estos Temas en dos categorías. Por un lado están los Temas que se identifican con grandes valores tradicionales, aunque presentados desde una perspectiva actual. Por otro lado, están los Temas que se relacionan con aspectos utilitaristas y concretos de nuestro mundo.

- Los Temas que se identifican con grandes valores tradicionales son: la Educación moral y cívica, asociada con valores éticos y de solidaridad ciudadana; la Educación para la paz, que se identifica con valores democráticos y de tolerancia; y la Educación para la igualdad de oportunidades entre los sexos, que se relaciona con valores de igualdad y justicia. Los valores citados representan aspiraciones muy antiguas de los seres racionales y se identifican con una vida auténticamente humana. Los Temas relacionados con ellos aluden a problemas que han estado presentes en la historia de la humanidad a través de las distintas épocas.

- El resto de los Temas Transversales (Educación sexual, Educación ambiental, Educación del consumidor, Educación para la salud, y Educación vial) representan problemas coyunturales al mundo actual.
Estos Temas Transversales vinculados con la actualidad, no identifican su finalidad con valores específicos, aunque a través de ellos puedan desarrollarse gran parte de los valores comentados. Su finalidad se asocia con una mejora de calidad de vida, una vida que para plantearse estas problemáticas tiene que haber alcanzado un determinado nivel de desarrollo, por lo que podemos decir que aluden a problemas de nuestra sociedad que tendrían poco sentido en otras épocas o sociedades.

González (1994), es consciente de que los valores éticos no tienen mucho espacio en la sociedad actual y poniéndose en el lugar del profesorado se pregunta cómo hacer posible la educación en unos valores que con frecuencia no se reflejan en nuestra realidad social cotidiana. La respuesta que ofrece es que, ante esta situación e incidiendo en los signos de esperanza que también se producen dentro de ella, urge plantear y desarrollar posiciones éticas alternativas fundamentadas en la educación que lleven al renacimiento de un humanismo nuevo que ha de suponer un cambio profundo en las actitudes personales y una nueva forma de habitar el planeta.

Yus (1997), declara que el concepto de transversalidad se asimila a una dimensión de comprensión social, e involucra aquellos conocimientos, valores y actitudes que se requieren para el desarrollo de comportamientos que le permita al estudiante actuar en el medio social y natural. Se orienta por tanto, hacia la comprensión de la sociedad a través de formas de ver, de juzgar y actuar de manera que se incentive la convivencia social, democrática e intercultural. Para este autor la inclusión en el currículo de los Temas Transversales representa un adorno moral para una sociedad que pretende ser humanista, pero que por encima de todo valora el desarrollo de las áreas relacionadas con la producción.

Para Moreno (1993), los aprendizajes en la educación infantil y la enseñanza obligatoria no deben tratar de conseguir la especialización en materias disciplinares, sino que en estas etapas educativas las áreas deberían ser medios o instrumentos para conseguir. 
En realidad, las finalidades que se persiguen con los llamados Temas Transversales estarían relacionados con la auténtica reforma educativa; pues desde un modelo de interpretación histórico, define los Temas Transversales como finalidades educativas fundamentadas en las necesidades que desde la esfera privada transcienden a la esfera de lo público rompiendo con la tradición de las disciplinas clásicas que dieron origen a nuestra cultura.

La problemática que plantea la transversalidad, según estos autores, no es algo que se pueda reducir a un problema de currículo y aprendizaje, sino que está engranada en otros mecanismos, la mayor parte ocultos que la condicionan.

Desde este análisis los Temas Transversales suponen unos valores que no sólo entrarían en conflicto con numerosas fuentes de emisión de mensajes, sino que pueden entrar en conflicto con los propios valores del profesorado $\mathrm{y}$, desde luego, con la propia estructura organizativa de la institución educativa. Por lo tanto, los principios y valores resultan ser mediadores del proceso educativo a los proyectos en acción.

El tema de la transversalidad en la educación es un paradigma que las Instituciones de educación deben tomar con mayor seriedad; el desafío no radica en la implementación de nuevos diseños curriculares o los retos de comprensión de fenómenos complejos o multidimensionales; sino la necesidad inmediata de transmitir en el alumno contenidos socializadores (valores, normas y actitudes). Esto implica incorporar la parte afectiva en el proceso de aprendizaje; en un plano metodológico; es decir, implementar en los programas la incorporación de los ejes transversales institucionales en los diseños de curso.

No se trata de transversalizar todos los objetivos y contenidos del diseño curricular, pero sí de realizar una selección consciente de los mismos, con el criterio de entender cuáles son los más aptos y proceder a realizar la transversalización como lo señala Reyzábal (1995).
La transversalidad tienen gran importancia en la Educación Superior, radica en pronunciarse decididamente por una acción formativa integral, que contemple de manera equilibrada tanto los aspectos intelectuales como los morales y que potencie el desarrollo armónico de la personalidad de los alumnos y alumnas, sin olvidar el problemático contexto social en que ellos viven. Para De la Vega (2012), La transversalidad es un enfoque dirigido al mejoramiento de la calidad educativa, buscando superar la fragmentación de las áreas del conocimiento, a partir del desarrollo de una visión holística, que promueva además la aprehensión de valores, la formación de actitudes, la expresión de sentimientos, maneras de entender el mundo y a las relaciones sociales en un contexto específico. Todos estos elementos se asocian a la necesidad social de introducir las preocupaciones de la sociedad, en el diseño curricular y las prácticas educativas.

\section{Objetivo de la investigación}

Contribuir en la mejorara de la calidad educativa, asumiendo la formación del Ingeniero a través de su desarrollo integral con un enfoque transversal; que prepare al estudiante para enfrentar los retos de esta sociedad tan cambiante.

\section{Hipótesis}

El Ingeniero logrará un desarrollo integral a través de la transversalidad en los procesos de aprendizaje, para fortalecer el desarrollo de sus capacidades con las nuevas condiciones de trabajo y las formas de vida actuales, que no han sido suficientemente atendidas y que requieren solución inmediata.

\section{Metodología a desarrollar}

El método utilizado para este trabajo es el enfoque de sistemas que permite identificar y comprender con mayor claridad y profundidad los problemas que se plantean, sus múltiples causas, consecuencias y la solución de estos.

Para captar la información y cumplir con los objetivos de este trabajo se efectuaron tres encuestas: 
La primera encuesta se aplicó a los alumnos inscritos en los diferentes semestres de las carreras del Instituto Tecnológico de Puebla (ITP) del periodo enero-junio del presente año, de las ocho carreras del ITP: Ing. Logística, Lic. en Administración, Ing. En Gestión Empresarial, Ing. En TIC, Ing. Eléctrica, Ing. Electrónica, Ing. Mecánica, Ing. Industrial (10 encuestas por carrera).

La segunda, aplicada a los egresados; orientados a captar información acerca del grado de satisfacción de su formación profesional en el ITP y cómo ha impactado su preparación en su inserción y desempeño en el mercado labor y si ha cumplido con sus expectativa de plan de vida.

Para determinar el tamaño de la muestra se aplicó la siguiente fórmula:

$\mathrm{n}=\frac{Z^{2} p(q)(N)}{N e^{2}+z^{2} p(q)}$

Se estableció como nivel de confianza el $95 \%$ y un error del $5 \%$. egresados.

La población a estudiar es de 1672

Valores a estimar.

$$
\mathrm{n}=324
$$

$$
\mathrm{e}=5 \%=0.05 \text { o } 10 \%=0.1
$$

$\mathrm{Z}=1.96$ tabla de distribución normal para el $95 \%$ de confiabilidad y $5 \%$ error $10 \%$ error.

$\mathrm{Z}=1.65$ para el $90 \%$ de confiabilidad $\mathrm{y}$

$$
\begin{gathered}
\mathrm{N}=2078 \text { (Población) } \\
\mathrm{p}=0.50 \\
\mathrm{q}=0.50 \\
\mathrm{n}=\frac{(1.96)^{2}(0.5)(1-0.50)(530)}{(530)(.05)^{2}+(1.96)^{2}(0.5)(1-0.50)}=\frac{50.902}{.2285}=222
\end{gathered}
$$

Por tanto, para que el estudio tenga un 95\% de confiabilidad el tamaño de muestra requerido fue de 222 egresados.
Las preguntas dirigidas a los alumnos inscritos actualmente; están orientados a captar información relacionada con el objetivo de esta investigación; van en función de su formación sociocultural:

- Relaciones interpersonales.

- Uso de las TIC.

- Actividades recreativas y artísticas.

También se realizó una encuesta dirigida a los docentes para conocer el desempeño del alumno en el aula: cumplimiento, participación, actitud, valores, desarrollo de habilidades cognitivas, metacognitivas, motivacionales; factores que contribuyen a fortalecer sus procesos personales de armonía interior y satisfacción personal, importantes para conocer y aplicar estrategias en dónde el alumno logre su desarrollo personal, que le permita mantener un estado de bienestar y un ambiente de aprendizaje apropiado, debido a que, a pesar de los avances y logros del sistema educativo nacional, se han acentuado estos factores que impactan negativamente la permanencia y rendimiento escolar del alumno. Cabe mencionar que algunos resultados de esta encuesta ya se habían expuesto en el documento"La vinculación como medio para mejorar el desempeño profesional y lograr el desarrollo humano integral del ingeniero" como continuación a este trabajo de investigación, mencionado anteriormente.

\section{Resultados}

Resultados obtenidos de los alumnos inscritos en los diferentes semestres de las carreras del ITP, con la finalidad de obtener datos más claros sobre su desarrollo integral.

\section{Relaciones interpersonales}

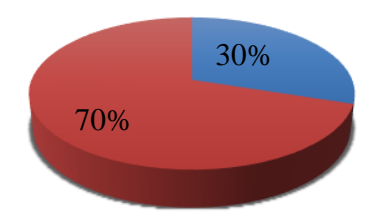

- Convive con familia $\quad$ Convive con amigos

Gráfico 1 Relaciones interpersonales 
El Gráfico 1, representa claramente que hay una problemática en las relaciones interpersonales de los alumnos, debido a que el $70 \%$ convive más tiempo con sus amigos; el $30 \%$ pasa más tiempo conviviendo con su familia que con sus amigos; la justificación es que la mayoría son compañeros de escuela y sigue la comunicación por medio del teléfono móvil. Está claro que en muchas ocasiones, el adolescente prefiere pasar largos períodos de tiempo frente a la computadora o el celular, que compartir una buena plática con sus padres, hermanos o amigos; incluso estando presente en las reuniones el adolescente puede encontrarse literalmente "perdido" en las redes sociales.

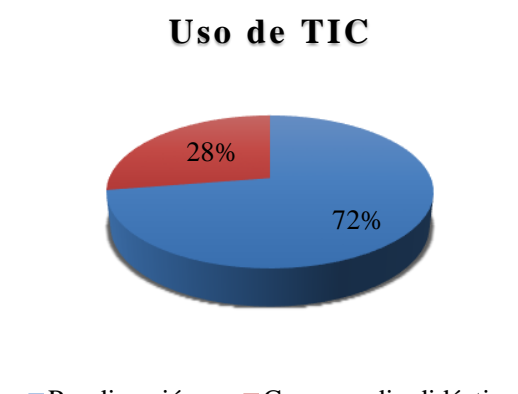

Gráfico 2 Uso de las TIC

Otro dato importante es el uso de las TIC de acuerdo a la pregunta ¿Para qué utiliza las TIC'S?, el $72 \%$ contestó por diversión, únicamente el $28 \%$ respondió que como medio didáctico, aunque esto no queda claro para ellos, ya que estas tareas no tienen la calidad que le podrían dar con las herramientas didácticas que les ofrecen estos medios; las TIC las utilizan para mirar videos, escribir o leer emails, bajar música; o se pasan el tiempo participando en juegos, o conectados a las redes sociales.

\section{Act. Recreativas y artísticas}

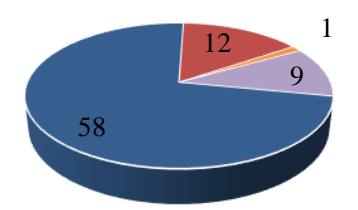

- Lee libros y va a la cine

- Asiste al teatro, exposiciónes o museos

- Practica algún deporte

- No realiza ninguna actividad

Gráfico 3 Actividades recreativas y artísticas
Con respecto a las actividades recreativas y artísticas que los alumnos desarrollan; de los 80 alumnos encuestado, sólo 12 leen libros y van al cine; 1 asiste a teatro o museos, 9 práctica algún deporte y lo delicado de la situación es que el 73 de ellos no realiza ninguna de estas. Las Instituciones de Educación Superior deberán promover eventos artísticos, en los que se integre a los alumnos; como participante o como organizador. De la encuesta aplicada a los egresados de las ocho carreras del ITP se obtuvieron los siguientes resultados:

\section{La carrera profesional le proporcionó} comptencias de liderazgo

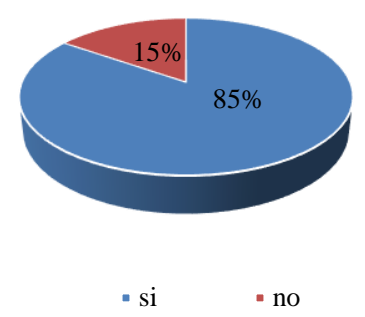

Gráfico 4 Expectativas de la carrera con su plan de vida

Según los resultados obtenidos nivel de satisfacción de su carrera profesional, el $85 \%$ de los egresados cubrió sus expectativas de plan de vida y se encuentran laborando en empresas con puestos en donde desarrollan liderazgo; el $15 \%$ restante manifestó que los empleos que ha tenido son eventuales y de menor responsabilidad.

Los resultados que manifiesta la OECD (2019) en su Resumen Ejecutivo nos dice que, hay datos representativos para evaluar las competencias de los egresados en México, algunos signos apuntan a unos niveles insuficientes de competencias, tanto Transversales como de disciplinas específicas.

Incrementar la calidad de la educación superior ha sido una prioridad de las políticas públicas desde hace mucho tiempo, pero los resultados son desiguales. 


\section{Dominio del idioma inglés}

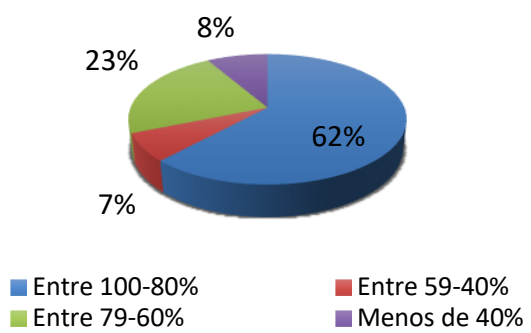

Gráfico 5 Dominio del idioma inglés

En este rubro, únicamente el $62 \%$ de los egresados dominan entre el 100-80\%, idioma inglés, el 23\% lo domina entre el 79-60\%, el resto se encuentra en un nivel muy bajo, aun cuando es un requisito cumplir con el curso en el ITP; los jóvenes al egresar deben cumplir con el nivel B1 intermedio 3 requisito para la titulación, es importante mencionar que el $68 \%$ no está titulado; un dato muy importante que influye en la calidad de la educación en México, que pasó del lugar 44 al 57 en el dominio del inglés a nivel mundial. De acuerdo con un estudio de la empresa educativa internacional Education First (EF), los resultados anuales del Índice de Dominio (EPI por sus siglas en inglés), son el único indicador mundial que mide el nivel de inglés de la población no angloparlante.

Según EF, los mexicanos solo pueden mantener una conversación básica, redactar un email y recorrer como turista una ciudad de habla inglesa.

A nivel Latinoamérica, el resultado sigue sin ser alentador, pues México se encuentra en la novena posición, superado por 1. Argentina, 2. Costa Rica, 3. República Dominicana, 4. Uruguay, 5. Chile, 6. Brasil, 7. Guatemala y 8. Panamá. Debido a esta problemática el $72 \%$ de los profesionistas pierden una oportunidad laboral.
En el caso de la encuesta aplicada a docentes, se obtuvo que el área de oportunidad más relevante se centra en el desempeño de habilidades y actitud del alumno, esto provoca en él barreras que le impiden crear aprendizaje; el profesor debe estar capacitado para evaluar la magnitud y dificultad de dichas barreras; por lo anterior, debe mantener una constante capacitación y desarrollar competencias para acompañar y valorar al estudiante en un marco contextual real, de esta manera deberá influir motivado para enseñar y colaborar en su formación, el objetivo de esta práctica en clase es despertar el interés del estudiante y promover su autoconocimiento personal en relación con la toma de conciencia de los factores que le impulsen a crear su propio proceso de aprendizaje.

\section{Conclusiones}

Para mejorar la calidad educativa se quiere un cambio en los procesos de aprendizaje del alumno, lo que implica una transformación de las funciones $\mathrm{y}$ tareas educativas que se desempeñan tradicionalmente, la transversalidad contribuirá a la formación del espíritu investigador y emprendedor (docentealumno), en el desarrollo de habilidades cognitivas, metacognitivas, motivacionales, que contribuyan a fortalecer en él sus procesos de armonía interior y satisfacción personal en la práctica y la reflexión individual.

Se ha mencionado ya el rol del profesor tan importante para que el alumno logre relaciones interdependientes de sus experiencias, su función debe basarse en la confianza, el esfuerzo conjunto y la comunicación. En los modelos de enseñanza tradicional suelen construirse algunas barreras derivadas de las sensibilidades, estas pueden ser de tipo interculturales, creencias, habilidades del lenguaje etc. Por esta razón y aun cuando el aprendizaje debe estar centrado en el alumno; el profesor debe estar capacitado para evaluar la magnitud y dificultad de dichas barreras; para tal fin debe desarrollar competencias para acompañar y evaluar a sus estudiantes en el marco de una aplicación contextualizada y real. 
Así mismo es de vital importancia realizar un análisis para mejorar la calidad educativa centrada en el docente; el cual, debe diseñar estrategias de acción para el desarrollo de sus actividades, que promueva el pensamiento crítico y creativo sobre su tarea; que programe tutorías y se mantenga en constante a la vanguardia para incorporar herramientas tecnológicas en su práctica docente.

La tecnología representa un importante avance para el ser humano, mejora la comunicación y permite estar informados en tiempo real, sin embargo no debe olvidarse que este no sustituye las relaciones persona-persona que todos necesitamos tener. Estos espacios virtuales ofrecen a la juventud la oportunidad de estar en contacto con otras personas de su edad, incluso en otros países, sin embargo, estar inmersos en un mundo exclusivamente virtual provoca en muchas ocasiones el aislamiento social en la juventud. La solución sería que las Institución educativas incluyan una materia sobre herramientas didácticas para que el alumno desarrolle sus capacidades y habilidades creativas y de innovación; que le permita acceder a la información a partir de fuentes digitales; a dar prioridad a las imágenes en movimiento y a la música por encima del texto; a sentirse cómodos realizando tareas múltiples simultáneamente y a obtener conocimientos procesando información discontinua y no lineal.

\section{Referencias}

De la Vega, L. (2012). Currículum y objetivos fundamentales transversales en Chile: resultados y proyecciones. Akademéia revista digital Universidad UCINF, 3 (1). Recuperado de http://www.revistaakademeia.cl/?p=740

Díaz F. y Hernández, G. (2010). Estrategias docentes para un aprendizaje significativo $\left(3^{\mathrm{a}}\right.$. Ed.). México: McGraw-Hill

Education, OECD Publishing, Paris. EF EPI EF English Proficiency Index.Empresa educativa internacional Education First (EF). Recuperado de: https://doi.org/10.1787/9789264309432

https://www.ef.com.mx/epi/regions/latinamerica/mexico/
Moreno, M. (1993). Los Temas Transversales: una enseñanza mirando hacia delante. Los temas transversales. Claves de la educación integral, Madrid: Aula XXI, Santillana.

Muñoz, A. (1997). Los temas transversales del currículo educativo actual. Revista Complutense de Educación, vol. 8, n. 0 2,1997. Servicio de Publicaciones. Universidad Complutense. Madrid.

OCDE (2010) Acuerdo de cooperación MéxicoOCDE para mejorar la calidad de la educación. Recuperado de https://www.oecd.org/education/school/462167 86.pdf

OECD (2019), Higher Education in Mexico: Labour Market Relevance and Outcomes, Higher

Ramos, Y. (1997). Los valores: ejes transversales de la integración educativa. Colombia: Conveno Andrés Bello.

Reyzábal, M., Sanz, A. (1995). Los ejes transversales. Los ejes para la vida. Madrid. Editorial Escuela española

Rosales, C. (2015). Evolución y desarrollo actual de los Temas Transversales: posibilidades y límites. Foro de Educación, 13(18), pp. 143-160. doi: http://dx.doi.org/10.14516/fde.2015.013.018.00 8 